\title{
Study on the Role and Method of Print Texture Language in Shaping Spatial Emotion
}

\author{
Zheng Feng \\ School of Design, South China University of Technology, Guangdong, Guangzhou, 510006
}

Keywords: print texture language; shaping spatial emotion; modeling notation

\begin{abstract}
In recent years, the demand for the design of modern space has risen from material needs to spiritual needs. The new relationship between human and space has become a new demand. According to the work experience, this paper summarizes the role of print texture language in shaping spatial emotion, puts forward three reference methods shown in the print screen vision, print texture techniques and the semantic combination between mechanisms of material and modeling notation, and discusses the main methods of print texture language in shaping spatial emotion.
\end{abstract}

\section{Introduction}

Space is created mainly to meet people's demand. In the process of long-term evolution, space has different meanings for human survival in different times. The early physical space has gradually evolved into a multi-functional space now. With the constant change of demand, people have also realized innovation in the manufacture of space environment. In the design of modern space, in order to express emotional atmosphere well, the emotional and cultural care needs to be timely added to serve the people's life better.

\section{The Role of Print Texture Language in Shaping Spatial Emotion}

In the process of design of traditional space, the limitation of art and science makes texture language too single and its space extremely monotonous, which has a great influence on the development of spatial design thinking. By texture creation of print, spatial expression can realize its effective development to make the texture design more inspiring and thinking. Especially in the design of print art, the narrative and emotion of spatial texture are increased to endow more imaginary space about spatial texture for the masses.

First of all, the form of the texture is how the imprint is to be presented. The inprint is mainly processed by different plate making methods to determine the kind of print, then to install the plate and texture to the layout of a printed sheet for reflecting the relatively obvious changes of texture according to the actual demand. After the original version is determined, the artistic language of texture will be completely manifested after being processed by the corresponding printing techniques. The texture of the original version is mainly concerned with the texture of the plate of itself and the artificial texture of the later stage, which succeeds in presenting the texture of the print together. But actually the two are not independent during the process of shaping emotional space, and superposed by some specific pattern to form certain effect conveyed by the images as to improve its tension performed by the whole picture's composition to manifest the features of print's texture. Therefore, the forms of the print's texture mainly include the following aspects: the performance of the plate's texture, the performance of artificial texture and the performance of the texture in the form of the picture ${ }^{[1]}$.

As for the primary texture form of the space, the way of direct placement is traditionally dominated. And for the natural texture, the visual aesthetic perception of the spatial sense can be reflected by the texture's features to generate the correspondent psychological effect. The form of the second texture can mainly divided into two main categories. The first is to reprocess it on the base of the primary texture, and then put it into the space after being polished. Or the new material 
can be directly explored, which has little relation with the primary texture; the texture of new material can be directly created by human.

In terms of the contrast the print's texture language and the emotion shaping mechanism of spatial texture, there are great differences in the forms of texture of the two, which also has a great influence on the shaping of texture. According to the emotional function, the way of emotional shaping can be concluded reasonably: whether print or texture, when to select the mode of production, the texture's attribute of the material should be firstly contemplated, especially the physical attribute of the texture has influence on the its semantics and the techniques of the texture itself, which will cause big difference in perceptual effects and modelling symbols. Generally speaking, the expression of the texture's emotion is mainly composed of its own semantics' conveying, expressing form, technique and so on. Although the two have the same perceptual expression and semantic communication, the form and attribute of the single print texture and the spatial texture are different. Therefore, in the process of emotional shaping, there are many different forms and texture attributes integrated into. And as for the sensuous aspect, they both can generate visual stimulation for the audiences. Then the audience in the space can also feel the texture by touching, so as to get the attribute information like softness and hardness, which will make the texture's performance more comprehensive.

In terms of spatial emotion expression, the prints texture can have an effect on spatial texture from the senses and the reflection level. In the senses level, the visual expressive force of print can be enhanced because the prints texture has substantial texture production ways, which including the visual form and features formed by the prints texture, such as "black and white shadow" "concavity" and so on. In addition, prints texture has more advantages in texture language, including texture techniques and picture visual effects and so on. Therefore, we can make texture techniques and picture visual effects as the main form of visual representations to deepen the performance factor of spatial texture effectively. On the sense level, print texture can form reasonable reference to the spatial texture, and on the reflection level, both the texture and spatial texture of prints have fully explored and applied "comprehension" and give play to the natural semantic effect of the material combined with its own fabric texture characteristics. ${ }^{[2]}$

\section{Main Methods of Texture Language in Shaping Spatial Emotion}

Firstly, we can use the changing between light and shadow of "black and white" level caused by texture gradients. In the process of the texture modeling, it can be presented in the form of fine particles or flake and then structure bulk texture wall properly. The resulting texture had better have reflectivity in nature, thus could be distinguished easily in different gradient, such as metal, wood texture and so on. We can also use the same texture repeatedly after the theme design is determined to make the received light has big different level and formed the pictures in two gray into a texture of change effect in black, white and grey. Secondly, the "black and white" level of light and shadow change can be caused by texture permeability. In addition to light angle, we can also project the material texture on different levels to produce light levels. For example, Pakistani artists designed a ceiling-mounted large light used the carved and arabesquitic board, and put it into a square room and project different figures onto the six walls around, thus forming special optical effects.

Wood texture is most commonly applied in the process of express texture technique. The wood has some natural grain texture which will leave some imprint in the later production of wood texture techniques, this has also been applied to many theme creation. Moreover, there are many artificial texture methods in xylograph with different cutting tools and marks. In the process of object description, knife marks can be used to coordinate the light and dark ash effectively so as to realize concrete description. The simple points, lines and surface can be used to express the abstract speech. Generally speaking, wood is often used on the ground, wall and other decoration, mainly for ornament and decoration. However, the use of large areas is obviously unrealistic as the high cost of manual engraving, but the small size of the engraving technique still obtains a lot of applications. Common forms of wood texture techniques include: partial implantation of artificial marks, and utilization of natural wood texture ${ }^{[3]}$. 
In the space design, the choice of texture can be combined with modeling symbols to realize the co transmission of semantics. Texture selection also needs to consider the coordination and cooperation between itself and other texture. It is necessary to make a reasonable building of the wall with the leading effect after the space style is determined, the print texture and modeling method are utilized to find the fit point of shape texture and material then the most emotional effect of the texture wall is designed. Similar to wood block's materials and modeling, it mainly involves the craft and language characteristics of texture in the process of express spatial texture and modeling symbols. When the modeling symbol is established, it is necessary to show the effect reasonably according to the physical characteristics of the material. For example, in the design of the reception desk background wall of the London International Language College, corrugated cardboard materials have been used to form a wavy shaped wall after cutting. It is easy to cut out the radians as the plasticity of paper is strong. As far as the material characteristics of corrugated paper are concerned, it is easy to touch the fluctuation of corrugated paper, thus a kind of approachable feeling is acquired.

\section{Summary}

In conclusion, the convey of texture language is mainly from the selection of plates. There is a big difference in panel's semantics as the physical properties are different, which leads to the appearance of the modeling symbols. In the process of spatial texture emotion shaping, the instrumentality has to be developed reasonably and the primary texture utilization and secondary texture molding are used to produce a better visual sensory experience and the effective combination of sensory experience and modeling symbols is realized.

\section{Acknowledgment}

Project: 2016 National Art Fund “The song of the spring” (Grant No.20163136)

\section{References}

[1] Baohua Cui. “A Study on the Pluralistic Breakthrough of Print Language under the Background of Contemporary Culture” [J] Art Education Research, 2017(10):20.

[2] Lirui Yang. "Research on the Application of Print in Modern Poster Design” [J]. Art Panorama 2017(05):122-123.

[3] Liping Zheng, Binfan Niu. "Innovative Research on the Sense of Concavo-convex and Texture Effect of Traditional Print in Modern Illustrations” [J]. Art and Design (Theory), 2017,2(03):39-41. 